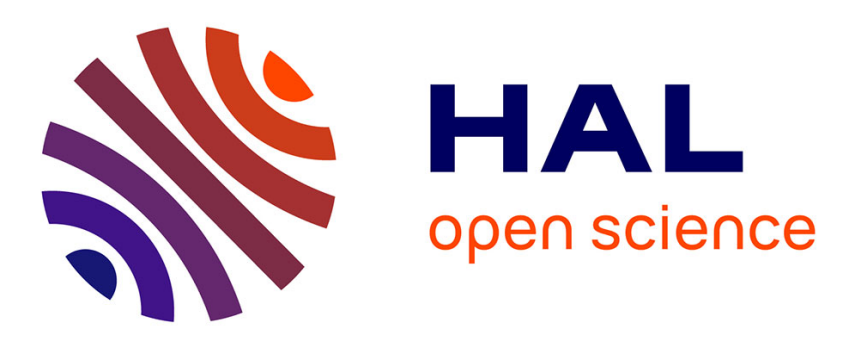

\title{
Simple and general method to calculate the dispersion properties of complex and aberrated stretchers-compressors
}

Frédéric Druon, Marc Hanna, Gaëlle Lucas-Leclin, Yoann Zaouter, Dimitris N. Papadopoulos, Patrick Georges

\section{To cite this version:}

Frédéric Druon, Marc Hanna, Gaëlle Lucas-Leclin, Yoann Zaouter, Dimitris N. Papadopoulos, et al.. Simple and general method to calculate the dispersion properties of complex and aberrated stretcherscompressors. Journal of the Optical Society of America B, 2008, 25 (5), pp.754-762. hal-00533396

\section{HAL Id: hal-00533396 https://hal.science/hal-00533396}

Submitted on 30 Mar 2012

HAL is a multi-disciplinary open access archive for the deposit and dissemination of scientific research documents, whether they are published or not. The documents may come from teaching and research institutions in France or abroad, or from public or private research centers.
L'archive ouverte pluridisciplinaire $\mathbf{H A L}$, est destinée au dépôt et à la diffusion de documents scientifiques de niveau recherche, publiés ou non, émanant des établissements d'enseignement et de recherche français ou étrangers, des laboratoires publics ou privés. 


\title{
Simple and general method to calculate the dispersion properties of complex and aberrated stretchers-compressors
}

\author{
Frédéric Druon, ${ }^{1, *}$ Marc Hanna, ${ }^{1}$ Gaëlle Lucas-Leclin, ${ }^{1}$ Yoann Zaouter, ${ }^{1,2}$ Dimitris Papadopoulos, ${ }^{1}$ and Patrick Georges ${ }^{1}$ \\ ${ }^{1}$ Laboratoire Charles Fabry de l'Institut d'Optique, CNRS, Université Paris-Sud, RD 128, 91127 Palaiseau, France \\ ${ }^{2}$ Amplitude Systemes, Pessac, France \\ *Corresponding author: frederic.druon@institutoptique.fr
}

Received November 19, 2007; revised January 21, 2008; accepted January 31, 2008; posted February 20, 2008 (Doc. ID 89872); published April 21, 2008

\begin{abstract}
We propose a general method to calculate the dispersion of an arbitrary optical system. It is based on a nonlinear extension of the $A B C D$ matrix model, where each optical element is described as an operator rather than a matrix. The deviation from a reference ray in terms of transverse position, angle, and phase as a function of wavelength is propagated through any optical system. This allows the calculation of all orders of dispersion, and also gives some insight in the space-time coupling phenomena such as spatial and angular chirp. This method is well-suited to compute the linear dispersive properties of complex and/or aberrated stretcher and compressor setups. (C) 2008 Optical Society of America
\end{abstract}

OCIS codes: $320.1590,260.2030$.

\section{INTRODUCTION}

Dispersion management in various ultrafast optics areas is becoming increasingly sophisticated, either to get shorter pulses at the right place in the experiment (e.g., the target), or manipulate pulses more accurately to achieve a very precise shaping (coherent control, ultrafast biophotonics). The use of active and passive dispersive elements to control pulse propagation is routine in ultrafast optics laboratories, for instance, in chirped-pulse amplification systems (CPA) [1]. The degree of control achieved on the pulse shape relies on how well the dispersion of these optical systems is characterized and designed. More specifically, with the recent explosion of the performances of ultrashort pulse fiber lasers and amplifiers, there is an increased need for precise dispersion control because the propagation lengths in these amplifying media is greatly increased compared to bulk systems. For basic arrangements such as gratings or prism pairs, analytical descriptions are possible. However, as an example, the optimization of a nonlinear fiber CPA system sometimes requires an independent control of second- and third-order dispersion [2], which is only possible through the use of more sophisticated arrangements. For these complex arrangements, for instance, hybrid prism-gratings sequences [3] or arrangements including advanced dispersive functionalities such as spatial light modulators, the introduced dispersion is generally calculated using general-purpose ray-tracing programs $[4,5]$ that are not specifically designed for the task. Furthermore, these programs only give numerical results and do not provide any physical insight into the problem. More convenient and simple methods are therefore sought after.

Previous attempts have been made at devising a method to describe the dispersive properties of optical systems such as linear matrix methods extending the
$A B C D$ model [6,7]. However, the linear character of these methods prevents one from accurately evaluating dispersion orders beyond the group-velocity dispersion, which become increasingly important as the pulse gets shorter and the amplifying medium gets longer. Recently, the coupling between spatial and temporal properties of a pulsed beam has been intensively investigated, both in terms of description of the physics involved [8] and the experimental description [9].

Here, we propose a general method to calculate the dispersion of an arbitrary optical system to arbitrary orders. It is based on an extension of the $A B C D$ matrix model, but is nonlinear, so that each optical element is described as an operator rather than a matrix. The deviation from a reference ray in terms of transverse position, angle, and phase as a function of wavelength is propagated through the system. This allows the calculation of all orders of dispersion, and also gives some insight into space-time coupling phenomena such as spatial and angular chirp. After a presentation of the general principle, we validate our method with well-known dispersive optical systems such as an ideal gratings and prisms compressor. The method is then applied to more complex systems, specifically a hybrid arrangement of gratings and prisms, and a grating compressor including aberrations effects.

\section{DESCRIPTION OF THE METHOD}

The method consists in following a reference ray through the optical system. We consider a single transverse dimension throughout the paper, the extension to two transverse dimensions is described in the final section. A vector containing the deviation from this reference ray in angle, transverse position, and optical phase shift as a 
function of wavelength is used to describe completely the beam at any position in the system:

$$
\vec{V}(\lambda)=\left(\begin{array}{c}
\delta \theta(\lambda) \\
\delta x(\lambda) \\
\delta \varphi(\lambda)
\end{array}\right) .
$$

An arbitrary wavelength-independent absolute phase shift can be added or subtracted from $\delta \varphi(\lambda)$ with no consequence on the physical problem. This vector is propagated using operators that are associated with the elements of the system (see Fig. 1). This model is therefore similar to the $A B C D$ matrix linear approach commonly used in ray and Gaussian beam optics. The addition of optical phase, in conjunction with the dependence of the vector coordinates on wavelength, allows the inclusion of dispersion properties and space-frequency coupling effects.

Let us first consider a plane interface with incident index $n_{1}$ and transmitted index $n_{2}$, as shown in Fig. 2. These indices can be wavelength dependent. This inter- face will be considered as infinitely thin so that no optical phase is accumulated along the reference ray, and no transverse shift is experienced at the interface. Moreover, this interface modifies the angle according to the law $\theta_{\text {out }}=f\left(\theta_{\text {in }}\right)$. This law depends on the nature of the optical component under consideration. This law is sufficient to determine the angle transformation $\delta \theta_{\text {out }}$. To determine the transverse shift transformation, simple geometric and trigonometric calculations are used to derive the intermediate distances labeled in Fig. 2: $x=\delta x_{\text {in }} \tan \theta_{0, \text { in }}, y$ $=x \sin \delta \theta_{\text {in }}, z=y / \cos \left(\theta_{0}+\delta \theta_{\text {in }}\right)$. The distance $\delta x_{\text {int }}$ along the interface is therefore given by

$$
\delta x_{\text {int }}=\delta x_{\text {in }}\left[\frac{1}{\cos \theta_{0, \text { in }}}+\frac{\tan \theta_{0, \text { in }} \sin \delta \theta_{\text {in }}}{\cos \left(\theta_{0, \text { in }}+\delta \theta_{\text {in }}\right)}\right] .
$$

A similar and symmetrical relationship between $\delta x_{\text {out }}$ and $\delta x_{\text {int }}$ can be obtained by considering the same distances in the output medium. The three components of the vector are therefore transformed as follows:

$$
\begin{gathered}
\delta \theta_{\text {out }}=f\left(\theta_{0, \text { in }}+\delta \theta_{\text {in }}\right)-f\left(\theta_{0, \text { in }}\right), \\
\delta x_{\text {out }}=\delta x_{\text {in }}\left(\frac{1}{\cos \theta_{0, \text { in }}}+\frac{\tan \theta_{0, \text { in }} \sin \delta \theta_{\text {in }}}{\cos \left(\theta_{0, \text { in }}+\delta \theta_{\text {in }}\right)}\right) /\left(\frac{1}{\cos \theta_{0, \text { out }}}+\frac{\tan \theta_{0, \text { out }} \sin \delta \theta_{\text {out }}}{\cos \left(\theta_{0, \text { out }}+\delta \theta_{\text {out }}\right)}\right), \\
\delta \varphi_{\text {out }}=\delta \varphi_{\text {in }},
\end{gathered}
$$

where $\theta_{0 \text {,in }}$ and $\theta_{0, \text { out }}$ are the input and output angle of the reference ray from the normal to the interface. All the angles are algebraic and are counted positive counterclockwise. This interface operator can be used to describe gratings, ideal prisms (assuming that they only change the angle and that the beam does not propagate through the prism medium), or simple plane interfaces between two media with different indices of refraction $n_{1}$ and $n_{2}$. For these elements the angle transformations $\theta_{\text {out }}=f\left(\theta_{\text {in }}\right)$ are given by

$$
\begin{aligned}
\theta_{\text {out }}= & \arcsin \left(\frac{n_{1}}{n_{2}} \sin \theta_{\text {in }}\right) \quad \text { plane interface, } \\
\theta_{\text {out }}= & \arcsin [n \sin (A \\
& \left.\left.-\arcsin \left(\sin \theta_{\text {in }} / n\right)\right)\right] \quad \text { very thin prism, } \\
\theta_{\text {out }}= & \arcsin \left[\frac{ \pm 1}{n_{2}}\left(\frac{-m \lambda}{d}+n_{1} \sin \theta_{\text {in }}\right)\right] \text { grating, }
\end{aligned}
$$

where $n$ is the prism refractive index, which varies with wavelength; the \pm 1 is positive for transmission gratings and negative for reflection gratings; $m$ is the grating order; $d$ is the groove spacing; and $A$ is the apex angle of a prism. These laws of transformation of the angles, along with the operators given above, allow the propagation of the beam through such optical elements.
Let us now consider freespace propagation through a medium with wavelength-dependent refractive index $n$. The operator is given by

$$
\begin{gathered}
\delta \theta_{\text {out }}=\delta \theta_{\text {in }}, \\
\delta x_{\text {out }}=\delta x_{\text {in }}+L \tan \delta \theta_{\text {in }}, \\
\delta \varphi_{\text {out }}=\delta \varphi_{\text {in }}+\frac{2 \pi n(\lambda) L}{\lambda} \cos \delta \theta_{\text {in }} .
\end{gathered}
$$

The phase difference seen by the beam at wavelength $\lambda$ along the reference beam is computed by evaluating the scalar product of the wave vector at $\lambda$ with the unit vector

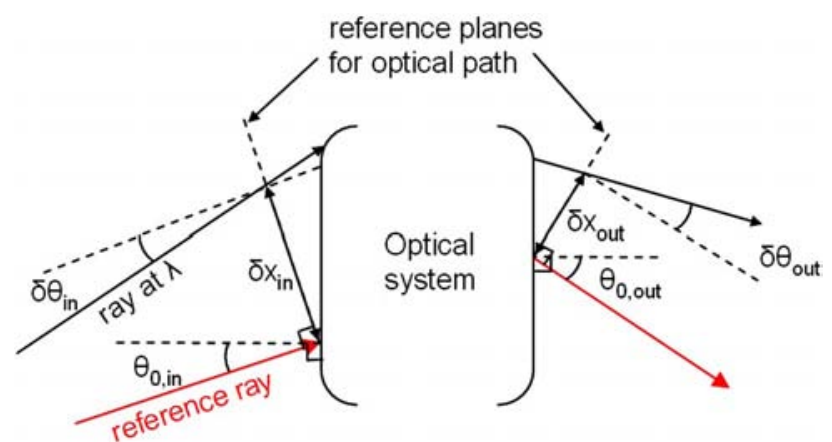

Fig. 1. (Color online) Geometry describing a single optical system. 


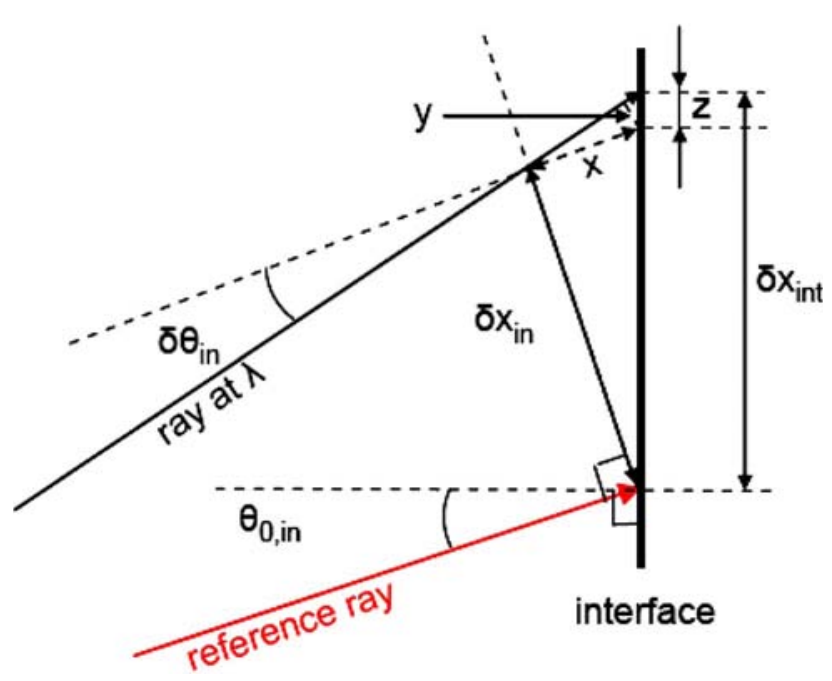

Fig. 2. (Color online) Geometry of the interface.

pointing in the reference beam direction. In combination with the previous operator describing angular interfaces, this allows the calculation of dispersion properties of the commonly used pairs of gratings or prisms used in compressors, and arbitrary arrangements of these elements. Although the prism formula described in Eq. (3) does not consider propagation in the material, it can be taken into account by using the following sequence of operators: plane interface-freespace propagation in glass-plane interface.

We now consider the description of mirrors and lenses that are used in stretcher setups. For a spherical interface or mirror with the reference ray on the optical axis, geometrical considerations similar to those used for the plane interface allow us to obtain the angle, transverse coordinate, and phase transformation:

$$
\begin{aligned}
\delta \theta_{\text {out }}= & a \sin \left(\frac{n_{1}}{n_{2}} \sin \left(\operatorname{sgn}\left(n_{2}\right) \cdot \delta \theta_{\text {in }}-a \tan \left(\frac{\delta x_{\text {in }}}{R}\right)\right)\right) \\
& +a \tan \left(\frac{\delta x_{\text {in }}}{R}\right), \\
\delta \varphi_{\text {out }}= & \delta \varphi_{\text {in }}+\frac{2 \pi}{\lambda} \frac{\left(n_{2}-n_{1}\right)}{n_{1}} R \\
& \times\left(\sqrt{1-\sin ^{2}\left(a \tan \left(\frac{\delta x_{\text {in }}}{R}\right)\right)}-1\right),
\end{aligned}
$$

where $R$ is the radius of curvature of the mirror interface (negative for a convex surface), $n_{1}$ is the incident medium refractive index, $n_{2}$ is the transmitted medium refractive index $\left(n_{2}=-n_{1}\right.$ for a mirror). The $\operatorname{sgn}\left(n_{2}\right)$ term in the angle equation must be added for mirrors because of the change of propagation direction. The term $\sin \left(a \tan \left(\delta x_{\text {in }} / R\right)\right)$ in the phase equation stems from the plane nature of our reference compared to the spherical surface under consideration. This operator can be used along with the freespace operator to describe any combination of spherical surfaces such as a spherical lens or more complex systems such as doublets. Since the freespace operator takes into account the refractive index dependence on wavelength, chromatic aberrations are readily included. Two special cases of spherical surfaces or a combination thereof are described now for convenience. For a spherical mirror in air with $n_{1}=1$ and $n_{2}=$ -1 , the operator is given by:

$$
\begin{gathered}
\delta \theta_{\text {out }}=\delta \theta_{\text {in }}+2 a \tan \left(\frac{\delta x_{\text {in }}}{R}\right), \\
\delta x_{\text {out }}=\delta x_{\text {in }}, \\
\delta \varphi_{\text {out }}=\delta \varphi_{\text {in }}+\frac{2 \pi}{\lambda} 2 R \sqrt{1-\sin ^{2}\left(a \tan \left(\frac{\delta x_{\text {in }}}{R}\right)\right)} .
\end{gathered}
$$

For a spherical plane-convex (concave) thin lens with focal length $f$, index $n$, set to properly focus a beam (plane interface towards focus), the following operator is obtained:

$$
\begin{gathered}
\delta \theta_{\text {out }} \approx \delta \theta_{\text {in }}-\frac{\delta x_{\text {in }}}{f}+\frac{\delta x^{3}}{f^{3}(n-1)^{3}}\left(\frac{5}{6}-\frac{1}{2 n}-\frac{n^{2}}{2}+\frac{n^{3}}{6}\right), \\
\delta x_{\text {out }}=\delta x_{\text {in }}, \\
\delta \varphi_{\text {out }} \approx \delta \varphi_{\text {in }}+\frac{2 \pi}{\lambda}\left[\frac{\delta x_{\text {in }}^{2}}{2 f}+\frac{3 \delta x_{\text {in }}^{4}}{32 f^{3}(n-1)^{2}}\right] .
\end{gathered}
$$

Arbitrary thick lenses can also be described using a combination of plane and spherical interfaces, and freespace propagation in various glass materials. Offset aberrations can be described by including $h_{0}$, the position of the reference beam relative to the optical center. This allows the description of the optimization of the lens position in stretcher setups:

$$
\begin{aligned}
& \delta \theta_{\text {out }}=a \sin \left(\frac{n_{1}}{n_{2}} \sin \left(\operatorname{sgn}\left(n_{2}\right) . \delta \theta_{\text {in }}-a \tan \left(\frac{\delta x_{\text {in }}+h_{0}}{R}\right)\right)\right) \\
& +a \tan \left(\frac{\delta x_{\mathrm{in}}+h_{0}}{R}\right)-\theta_{0, \mathrm{out}} \\
& \delta x_{\text {out }}=\frac{\delta x_{\text {in }}}{\frac{1}{\cos \theta_{0, \text { out }}}+\frac{\tan \theta_{0, \text { out }} \sin \delta \theta_{\text {out }}}{\cos \left(\theta_{0, \text { out }}+\delta \theta_{\text {out }}\right)}}, \\
& \delta \varphi_{\text {out }}=\delta \varphi_{\text {in }}+\frac{2 \pi}{\lambda} \frac{\left(n_{2}-n_{1}\right)}{n_{1}} R \\
& \times\left(\sqrt{1-\sin ^{2}\left(a \tan \left(\frac{\delta x_{\mathrm{in}}+h_{0}}{R}\right)\right)}-1\right),
\end{aligned}
$$

with

$$
\theta_{0, \text { out }}=a \sin \left(\frac{n_{1}}{n_{2}} \sin \left(-a \tan \left(\frac{h_{0}}{R}\right)\right)+a \tan \left(\frac{h_{0}}{R}\right)\right) .
$$

Aspherical optics can also be described using this method. For a general mirror with conic constant $\varepsilon_{a}$ and 
focal length $f$, the operators can be expanded in a Taylor series, giving the following result:

$$
\begin{gathered}
\delta \theta_{\text {out }} \approx \delta \theta_{\text {in }}-\frac{\delta x_{\text {in }}}{f}+\frac{\delta x_{\text {in }}^{3}}{f^{3}}\left(\frac{1}{3}-\frac{\left(1+\varepsilon_{a}\right)}{4}\right), \\
\delta x_{\text {out }}=\delta x_{\text {in }}, \\
\delta \varphi_{\text {out }} \approx \delta \varphi_{\text {in }}-\frac{2 \pi n}{\lambda}\left[\frac{\delta x_{\text {in }}^{2}}{2 f}+\left(\frac{1+\varepsilon_{a}}{4}-1\right) \frac{\delta x_{\text {in }}^{4}}{8 f^{3}}\right],
\end{gathered}
$$

where $\varepsilon_{a}=0$ corresponds to a sphere, $\varepsilon_{a}=-1$ to a paraboloid, $\varepsilon_{a}<-1$ to a hyperboloid, and $\varepsilon_{a}>-1$ to an ellipsoid surface.

Now that the mathematical transformation of the beam vector by optical elements is described, let us discuss the physical meaning of this vector. The first component, $\delta \theta(\lambda)$, is the angle as a function of wavelength, and is often referred to as the angular chirp or dispersion. This is basically the quantity on which gratings and prisms act. In traditional compressor arrangements, pairs of elements are used to get a zero overall angular dispersion. Some residual angular dispersion might be introduced due to chromatic aberrations of lenses, and this will be taken into account by our model. The second component, $\delta x(\lambda)$, is the variation of the space transverse coordinate as a function of wavelength, also called spatial chirp. This parameter can be used to determine the size of the optics. Moreover, typical arrangements use two pairs of dispersive elements to remove this spatial chirp, but aberrations in the system and misalignments might lead to nonzero spatial chirp at the output of the system. This effect can also be evaluated using our method. Finally, the third quantity, $\delta \varphi(\lambda)$, is the accumulated phase along the reference beam as a function of wavelength. Repeated differentiations of this phase with respect to angular frequency yield arbitrary orders of the dispersion, which is the first aim of this work. The dispersion might then be used to calculate the temporal profile of a pulse going through the system, assuming that nonlinear effects are negligible. The vector is a description of the beam that can be interpreted as plane waves with wavelength-dependent parameters. However, finite beam size effects would require the description of Gaussian beams instead of plane waves, and are not included in the model in its present form.

This model can be implemented in a semianalytical fashion very easily: each operator is defined analytically, but is implemented in a numerical code for convenience. When precise numerical results are needed for complex systems, this is the preferred method: given the complexity of the formulas obtained for complex systems, very little physical insight is lost by implementing the method using a numerical code where a discrete wavelength step is defined, and each operator and vector is a function of this discrete variable. Moreover, this numerical implementation can be made very conveniently by creating a library of operators corresponding to each type of optical system.

However, nothing prevents one from using this model in a fully analytic way. This can be very useful for specific cases, e.g., when approximations can be made, or when comparing small deviations of a system from an ideal known case. In this context, and in contrast with commercial software ray-tracing programs, analytic formulas giving physical insight into the problem can be obtained. An example of such an analytic treatment is given in Section 5 .

\section{VALIDATION FOR KNOWN SYSTEMS}

Let us now consider the simple example of a transmission grating pair compressor used at Littrow incidence. The schematic of this system is described in Fig. 3. In terms of operators and vectors, this system is described as

$$
\vec{V}_{\text {out }}=\bar{G}_{\theta} \circ \bar{F}_{L} \circ \bar{G}_{-\theta} \circ \bar{F}_{L^{\prime}} \circ \bar{G}_{-\theta} \circ \bar{F}_{L} \circ \bar{G}_{\theta}\left(\vec{V}_{\text {in }}\right),
$$

where $\bar{G}_{\theta}$ is the grating operator with a reference ray incidence angle $\theta$ and $\bar{F}_{L}$ is the freespace propagation operator over a distance $L$ in air. The propagation over $L^{\prime}$ between the two gratings pair has no effect in the case of a perfect system.

An analytic description is readily available for this type of grating compressor. The second- and third-order spectral phases introduced by this arrangement are given by

$$
\begin{gathered}
\phi_{2}=-\frac{\lambda^{3} Z}{\pi c^{2} d^{2}}\left[1-\left(\frac{\lambda}{d}-\sin \theta\right)^{2}\right]^{-3 / 2}, \\
\phi_{3}=-\phi_{2} \frac{3 \lambda}{2 \pi c}\left[1+\frac{\lambda}{d} \frac{\lambda / d-\sin \theta}{1-(\lambda / d-\sin \theta)^{2}}\right],
\end{gathered}
$$

where $d$ is the groove spacing, $c$ is the speed of light, and $Z$ is the orthogonal distance between the gratings. In our example, we use 1250 lines/mm gratings at Littrow incidence around the central wavelength $1045 \mathrm{~nm}$. The gratings separation is $L=1 \mathrm{~cm}$. Figures 4 (a) and 4(b) display the second- and third-order spectral phases obtained through our method and the analytical formula. The agreement is perfect, and therefore validates basic aspects of our model.

We now use our model to evaluate the second-order dispersion of a prism compressor. Analytical results are available for arbitrary prism sequences [10]. We will use the following approximate result to validate our model for a four-prism compressor:

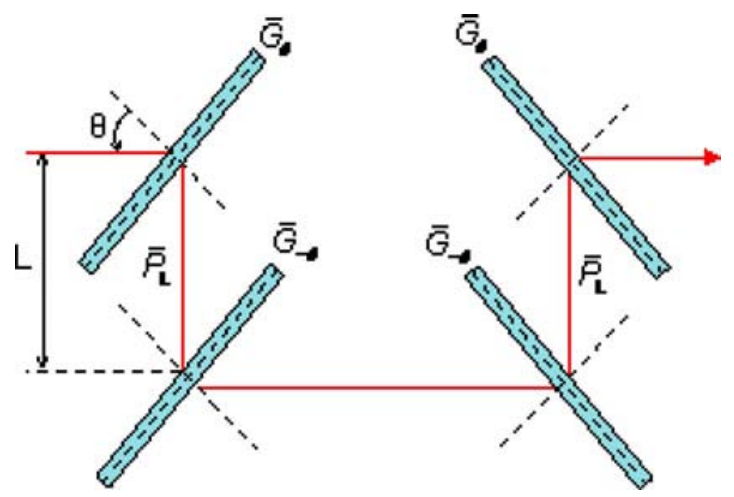

Fig. 3. (Color online) Transmission gratings compressor setup. 


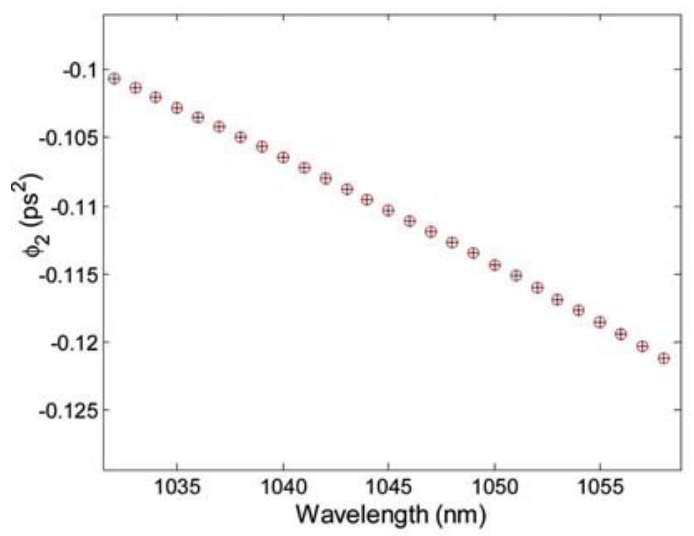

(a)

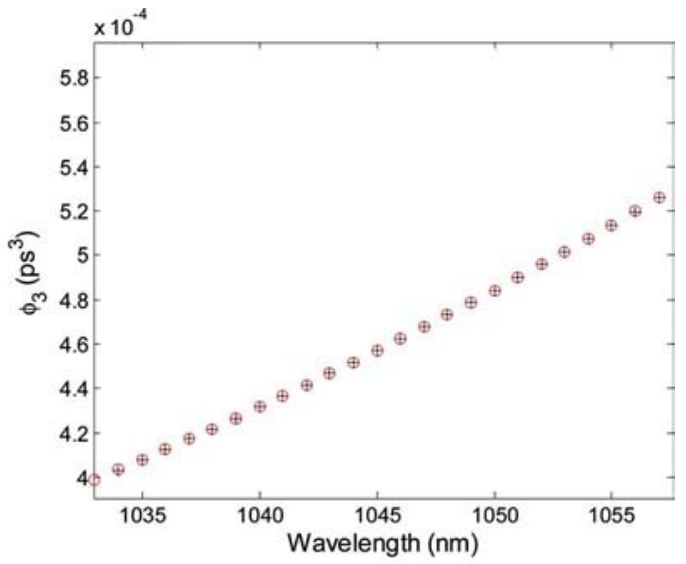

(b)

Fig. 4. (Color online) (a) Second-order spectral phase introduced by a gratings compressor as a function of wavelength. Black crosses, our method; red circles, analytic. (b) Third-order order spectral phase introduced by a gratings compressor as a function of wavelength. Black crosses, our method; red circles, analytic.

$$
\phi_{2} \approx-4 L \frac{\lambda_{0}^{3}}{\pi c^{2}}\left(\left.\frac{d n}{d \lambda}\right|_{\lambda_{0}}\right)^{2}+\left.L_{\text {prism }} \frac{\lambda_{0}^{3}}{2 \pi c^{2}} \frac{d^{2} n}{d \lambda^{2}}\right|_{\lambda_{0}},
$$

where $n$ is the prism material refractive index, $L$ is the distance between the apex of the prisms, and $L_{\text {prism }}$ is the distance traveled into the prism material.

We compare our method with the analytical approximation in two cases. First, we consider a system with no propagation in the prisms [Eq. (4)], second, we include the contribution of glass dispersion by propagating the beam inside the prism. In the latter case, the prism was modeled simply as two plane interfaces separated by a length of propagation in the glass. The wavelength dependence of the refractive index was obtained using a Sellmeier model for SF10. Figure 5 shows a good agreement of our model with the analytic prediction in both cases. When

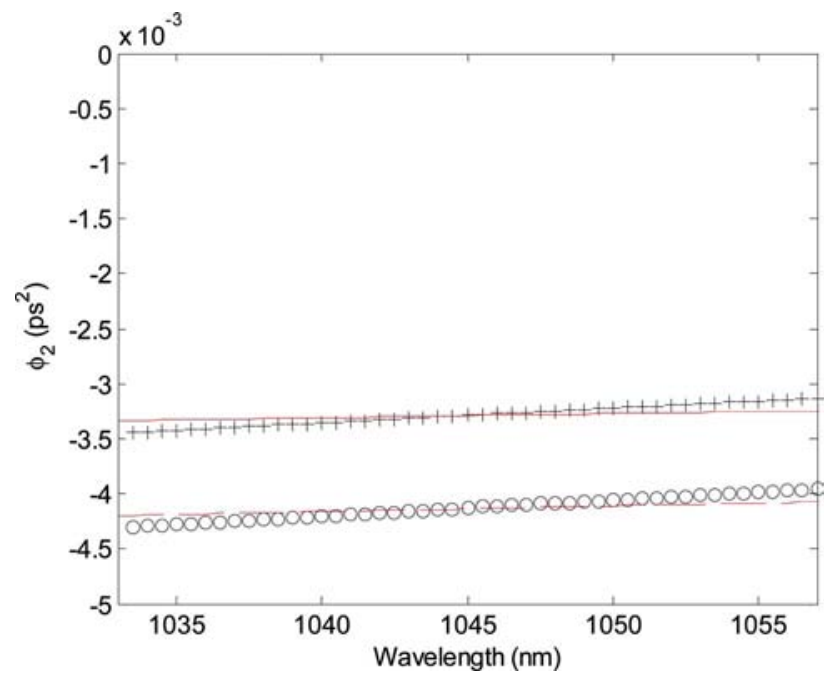

Fig. 5. (Color online) Second-order spectral phase introduced by a prism compressor as a function of wavelength. Circles: our method, no matter. Crosses: our method, $2 \mathrm{~mm}$ matter in prisms. Red dotted line: analytic approximation, no matter. Red solid line: analytic approximation, $2 \mathrm{~mm}$ matter in prisms. propagation in the bulk of the prisms is added, the expected rise of the second-order spectral phase is correctly predicted.

In this paragraph, only second- and third-order dispersions were shown, but nothing prevents the calculation of arbitrary orders of dispersion. In doing this, one must pay attention to the precision of the numerical differentiation by choosing the wavelength step appropriately.

\section{APPLICATION TO COMPLEX SYSTEMS}

We now apply our model to a complex dispersive system for which an analytical formula for the successive dispersion orders is not available. Our example is a combination of gratings, prisms, and lenses that was experimentally used to compensate the spectral phase introduced by a parabolic fiber amplifier [11].

Initially such a hybrid gratings-prims sequence compressor, known as a "grism," has been used in Ti:sapphire-based chirp pulse amplifier to compensate simultaneously second- and third-order dispersions in different CPA configurations $[12,13]$. Recently, grisms have been implemented in a femtosecond fiber oscillator leading to a record $33 \mathrm{fs}$ pulse generation [14], and in a fiber chirped-pulse amplification system [15]. Figure 6 shows a sketch of the grism configuration modeled here. The dispersion abilities of the two prisms arrangement is heightened by its incorporation into the gratings compressor. Thus, the adjustment of the distance $Z_{c}$ between the second grating and the image of the first one sets the rough

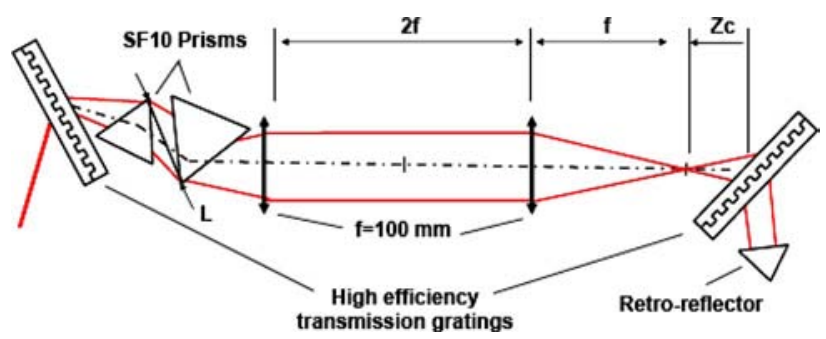

Fig. 6. (Color online) Hybrid prism-gratings compressor setup. 
compression of the parabolic pulses, while a careful choice of the distance L2 between the apexes of the two prisms adds the necessary third-order dispersion for a global optimal compression. We used our model to describe each optical element, fixed $\mathrm{L} 1=3 \mathrm{~mm}, Z_{c}=5.2 \mathrm{~mm}, f=15 \mathrm{~cm}$, and made L2 vary from 0 to $1 \mathrm{~cm}$. This allowed us to assess the $\varphi_{3} / \varphi_{2}$ ratio tuning range induced by the inclusion of prisms in the grating pair. Figure 7 shows this ratio obtained from our model as a function of the distance L2.

In the limit of $\mathrm{L} 2=0$, we check that the $\varphi_{3} / \varphi_{2}$ ratio takes the value for a simple gratings pair configuration. As the distance between prism apexes increases, the sign of the ratio changes as the third-order dispersion becomes dominated by the prism behavior, thereby allowing the system to compensate for propagation in bulk silica $\left(\varphi_{3} / \varphi_{2}=2 \mathrm{fs}\right)$. In our case, nonlinear propagation induced significant spectral phase, giving a target ratio of $3.5 \mathrm{fs}$ for the compressor, which was also achievable with the hybrid compressor. As a result, we achieved the compression of the fiber-amplified pulses down to $107 \mathrm{fs}$, compared to $127 \mathrm{fs}$ using a standard gratings compressor system [12].

\section{APPLICATION TO ABERRATED SYSTEMS}

A good example to test our model on systems including nonideal imaging optics is the comparison between the standard $f / 2 f / f$ stretcher and the Öffner stretcher [16,17] (Fig. 8), both with spherical mirrors and parabolic mirrors. The Öffner configuration is well-known to be aberration-free when used as a zero-dispersive line with spherical mirrors, which is not the case for the standard configuration. On the other hand, one can expect that, when replacing the spherical mirrors by parabolic mirrors, the classical stretcher becomes aberration-free, whereas the Öffner stretcher will exhibit aberrations. For this example we used a 1750 lines $/ \mathrm{mm}$ gratings with a central wavelength of $1030 \mathrm{~nm}$ at Littrow incidence, with $f=1 \mathrm{~m}$ mirrors.

The numerical results of our model are shown in Figs. 9(a) and 9(b). As expected, the perfect optics type for the Öffner stretcher is spherical, while the classical stretcher is perfect with parabolic mirrors. The spatial aberrations in the systems are also translated to the spectral domain, so that residual dispersion appears at the edges of the design bandwidth. Our model is able to predict such effects,

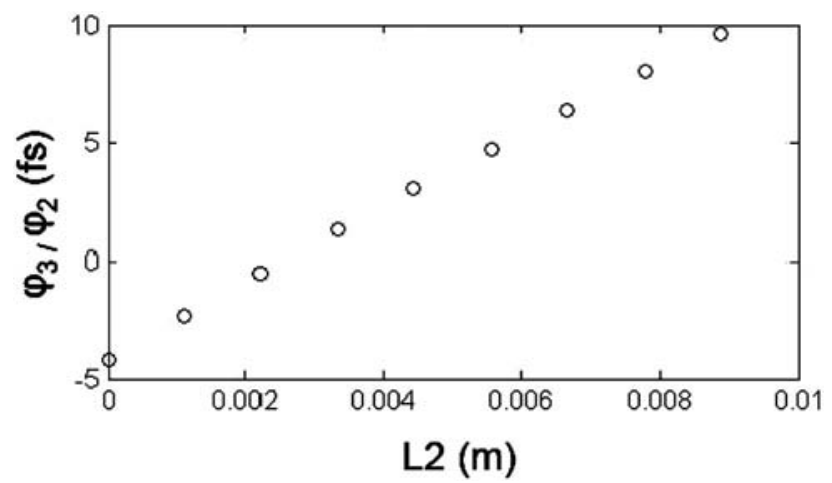

Fig. 7. Tuning of the third- to second-order spectral phase ratio as a function of the distance L2 (cf. Fig. 6).

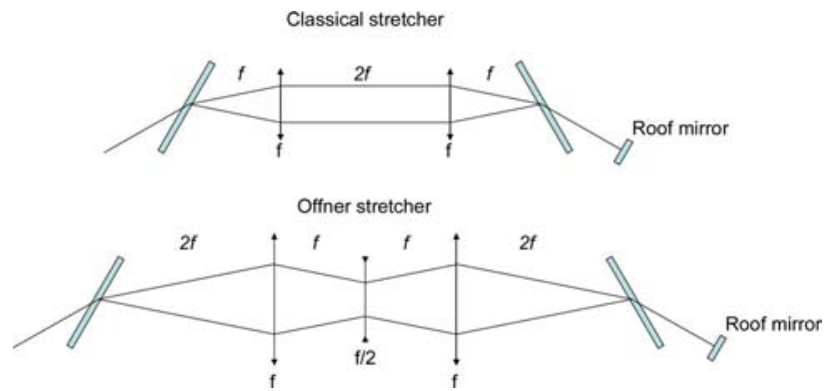

Fig. 8. (Color online) Two stretcher configurations.

which are very important when large beam diameters and bandwidth are considered, e.g., in high-energy ultrafast laser systems. This allows a precise design of compressors and the prediction of higher-order spectral phase distortions related to spatial aberrations.

To demonstrate the analytical use of our method, we have also calculated analytically the spectral phase added by nonparabolic aspherical optics described by Eq. (10) in the case of a standard stretcher. By following the propagation of the reference ray through the system and using the operators, along with symmetry arguments, this spectral phase is found to be

$$
\begin{gathered}
\varphi_{a}(\omega)=\left(1+\varepsilon_{a}\right) \frac{\omega}{c} f \tan ^{4} \delta \theta(\omega), \\
\delta \theta(\omega)=a \sin \left(-\frac{2 \pi c}{d \omega}+\sin \theta_{0}\right),
\end{gathered}
$$

where $\delta \theta$ is the angle deviation from the reference ray at the output of the first grating. This equation shows that the spectral phase correction is a fourth-order correction in terms of the angular dispersion imparted by the first grating. The parameters of a particular system (type of gratings, bandwidth considered), can be plugged into Eq. (10) to know the range of the additional spectral phase due to nonperfect optics.

To further illustrate the importance of aberrations in the design of stretchers-compressors, we consider a standard zero dispersion line with $f=1 \mathrm{~m}$ spherical mirrors, and 1100 lines/mm gratings at Littrow incidence, at the central wavelength of $1030 \mathrm{~nm}$. After modeling this system using our method, the obtained spectral phase is used to compute the output pulse characteristics when a Fourier transform-limited Gaussian pulse is fed to the line. We assume that the spectral intensity is completely unaltered. The results are shown in Fig. 10. The output pulse width is not modified for pulses longer than $100 \mathrm{fs}$. However, spatial aberration effects introduce a spectral phase that prevents the output pulse from being shorter than $80 \mathrm{fs}$. This can also be observed in the peak power of the output pulse normalized to the input peak power. For pulses longer than $100 \mathrm{fs}$ the reduction in peak power (sometimes denoted as the temporal Strehl ratio) is negligible, but becomes dramatic for broadband pulses shorter than $60 \mathrm{fs}$.

Although our examples aimed at specific systems, the presented model can be applied to study a wide range of dispersion effects including the dispersion modification 

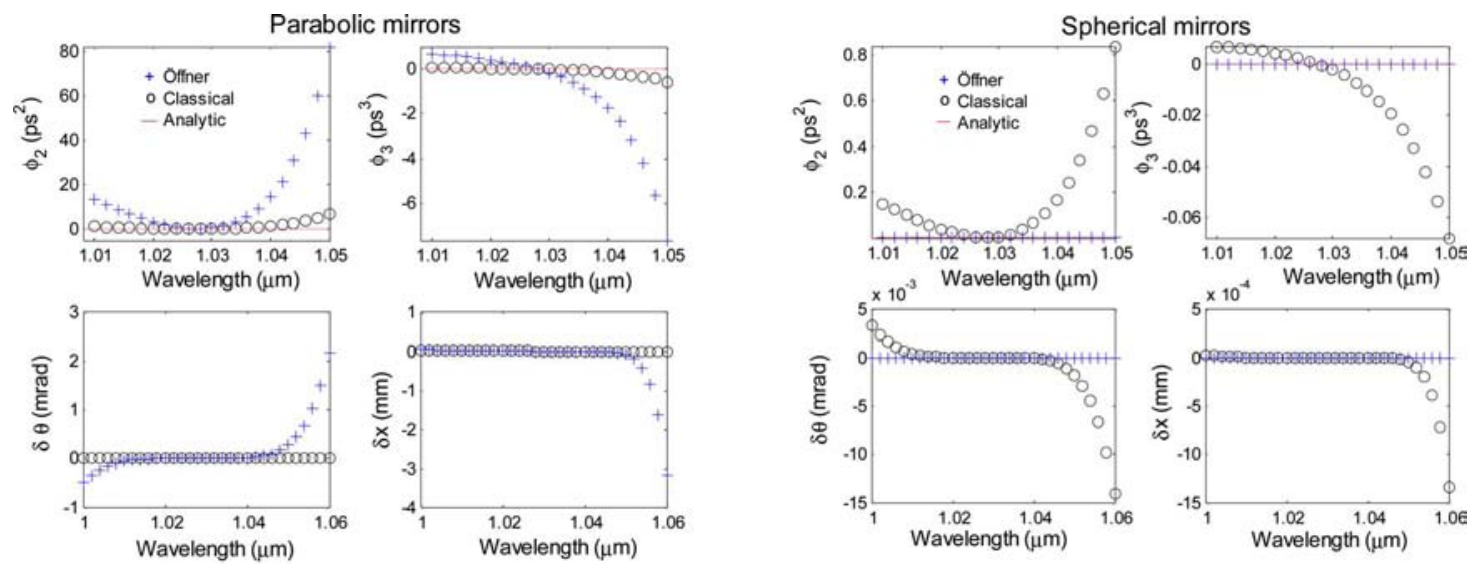

(a)

(b)

Fig. 9. (Color online) (a) Case of parabolic mirrors: second- (top left) and third-order spectral phase (top right), angular (bottom left) and spatial chirp (bottom right) introduced by a classical (black circles) and Öffner (blue crosses) stretcher. (b) Case of spherical mirrors: second- (top left) and third-order spectral phase (top right), angular (bottom left) and spatial chirp (bottom right) introduced by a classical (black circles) and Öffner (blue crosses) stretcher.

related to misalignment of optical components, dispersive lines including spatial light modulators, grism systems consisting of gratings directly written in prisms, and so on.

\section{MODEL EXTENSIONS}

Here are a few extensions that might be made to our model to adapt it to different design needs.
- Transverse bidimensionnal. As for the ABCD model, our method can take into account different propagation properties in the two sagittal and tangential directions by considering two separate sets of operators and vectors for each dimension. This extension can be used to take into account cylindrical lenses for example.

- Arbitrary surface optics. For an optical surface described by a two-dimensional function $Z$ of the transverse dimensions $\delta x_{\text {in }}$ and $\delta y_{\text {in }}$, the operator is given by

$$
\begin{aligned}
& \delta \theta_{\text {out }}= \delta \theta_{\text {in }}+a \sin \left(\frac{n_{1}}{n_{2}} \sin \left(\operatorname{sgn}\left(n_{2}\right) . \delta \theta_{\text {in }}-a \tan \left(\frac{\delta x_{\text {in }}}{R}-\frac{\delta x_{\text {in }}}{\sqrt{R^{2}-\delta x_{\text {in }}^{2}}}+\frac{\partial Z\left[\delta x_{\text {in }}, \delta y_{\text {in }}\right]}{\partial \delta x_{\text {in }}}\right)\right)\right) \\
&+a \tan \left(\frac{\delta x_{\text {in }}}{R}-\frac{\delta x_{\text {in }}}{\sqrt{R^{2}-\delta x_{\text {in }}^{2}}}+\frac{\partial Z\left[\delta x_{\text {in }}, \delta y_{\text {in }}\right]}{\partial \delta x_{\text {in }}}\right), \\
& \delta \gamma_{\text {out }}= \delta \gamma_{\text {in }}+a \sin \left(\frac{n_{1}}{n_{2}} \sin \left(\operatorname{sgn}\left(n_{2}\right) . \delta \gamma_{\text {in }}-a \tan \left(\frac{\delta y_{\text {in }}}{R}-\frac{\delta y_{\text {in }}}{\sqrt{R^{2}-\delta y_{\text {in }}^{2}}}+\frac{\partial Z\left[\delta x_{\text {in }}, \delta y_{\text {in }}\right]}{\partial \delta y_{\text {in }}}\right)\right)\right) \\
&+a \tan \left(\frac{\delta y_{\text {in }}}{R}-\frac{\delta y_{\text {in }}}{\sqrt{R^{2}-\delta y_{\text {in }}^{2}}}+\frac{\partial Z\left[\delta x_{\text {in }}, \delta y_{\text {in }}\right]}{\partial \delta y_{\text {in }}}\right), \\
& \delta x_{\text {out }}=\delta x_{\text {in }}, \\
& \delta \varphi_{\text {out }}=\delta y_{\text {out }}+\delta y_{\text {in }}, \\
& \lambda \frac{2 \pi n_{2}-n_{1}}{n_{1}} . Z\left[R \sin \left(a \tan \left(\frac{\delta x_{\text {in }}}{R}\right)\right), R \sin \left(a \tan \left(\frac{\delta y_{\text {in }}}{R}\right)\right)\right],
\end{aligned}
$$

where $\gamma$ is the angle in the other transverse dimension, and $R$ is the radius of curvature of the surface at the intersection with the reference ray.

- Optical intensity. Spatial and wavelength filtering effects might be included by adding an additional quantity $I$ in the vector describing the optical beam intensity, and additional operators to describe the amplitude transfer functions of optical elements: 


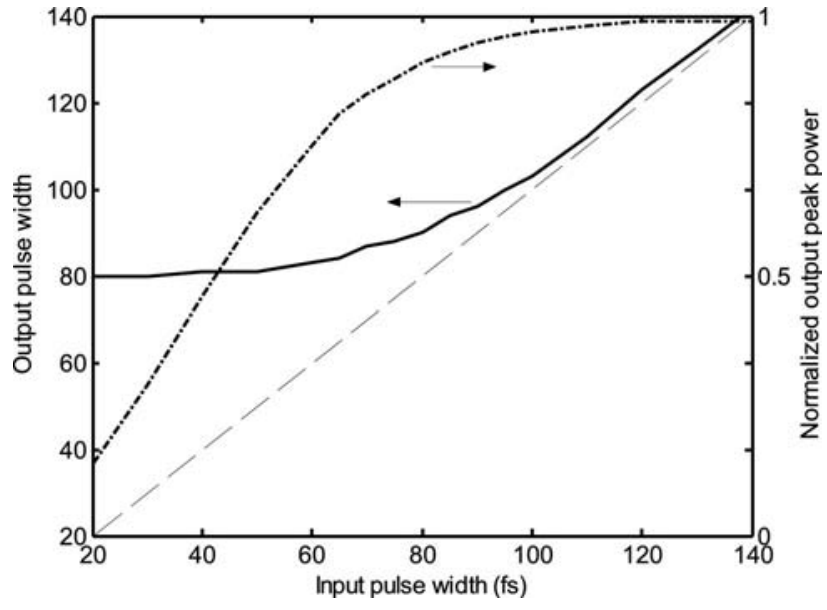

Fig. 10. Output pulse width and relative peak power as a function of input pulse width in a classical stretcher with $f=1 \mathrm{~m}$ optics, 1100 lines/mm gratings, and spherical mirrors.

$$
\vec{V}(\lambda)=\left(\begin{array}{c}
\delta \theta(\lambda) \\
\delta x(\lambda) \\
\delta \phi(\lambda) \\
I(\lambda)
\end{array}\right) .
$$

The transmission (reflection) characteristics and spatial clipping of the optics can easily be taken into account using the following equation:

$$
\begin{aligned}
I_{\text {out }}(\lambda)= & H\left(\delta x_{\text {in }}-h_{0}-\Phi / 2\right) \cdot\left(1-H\left(\delta x_{\text {in }}-h_{0}\right.\right. \\
& +\Phi / 2)) . T(\lambda) . I_{\text {in }}(\lambda),
\end{aligned}
$$

where $T$ is the transmission curve of the optics, $H$ is the Heaviside function, and $\Phi$ is the diameter of the optical element. If the input pulse is known, and assuming purely linear systems, the knowledge of both the spectral intensity and phase transmission of the system allows the complete knowledge of the output pulse shape.

- Active dispersive elements. Elements such as a Dazzler [18] or a spatial light modulator (SLM) can also be included in our model in a straightforward manner. The Dazzler simply introduces a predefined phase as a function of wavelength. An SLM imparts a user-defined spacedependent phase.

SLM:

$$
\begin{gathered}
\delta \theta_{\text {out }}=\delta \theta_{\text {in }}, \\
\delta x_{\text {out }}=\delta x_{\text {in }}, \\
\delta \varphi_{\text {out }}=\delta \varphi_{\text {in }}+\psi\left(\delta x_{\text {in }}\right), \\
I_{\text {out }}=T\left(\delta x_{\text {in }}\right) . I_{\text {in }},
\end{gathered}
$$

Dazzler:

$$
\begin{gathered}
\delta \theta_{\text {out }}=\delta \theta_{\text {in }}, \\
\delta x_{\text {out }}=\delta x_{\text {in }}, \\
\delta \varphi_{\text {out }}=\delta \varphi_{\text {in }}+\psi(\lambda),
\end{gathered}
$$

$$
I_{\text {out }}=T(\lambda) \cdot I_{\text {in }},
$$

where $\psi$ and $T$ are the phase and intensity modulation functions of the active system.

- Pulse front tilt. The inclusion of the group delay as a fourth vector component should also be possible. This component would correspond to the experienced group delay as a function of wavelength, and propagating this added quantity through the system would yield the pulse front tilt. Of course, this propagation requires an additional operator for each optical system that can be derived using geometrical arguments.

\section{CONCLUSION}

We have described a method to calculate all dispersion orders for arbitrary optical systems composed of freespace propagation in dispersive media, plane interfaces, gratings, prisms, lenses, and mirrors. The method is based on a vector approach where the beam deviation from a reference ray is followed through the system as a function of wavelength. Although basically analytical, the method is conveniently implemented numerically. We demonstrated its use for known systems such as gratings and prism pairs, and a more complex arrangement of gratings, prisms, and lenses that was used to optimally compress pulses from a parabolic amplifier. We also showed that it is possible to take into account spatial aberration effects in dispersive systems. This model represents a simple and powerful tool to design dispersive optical systems with information on chromatic and spatial aberrations and space-time coupling effects. This method can also be applied in a fully analytical way when small deviations from an ideal setup are examined, or when specific approximations can be made, thereby providing physical insight into complex dispersive systems.

\section{REFERENCES}

1. D. Strickland and G. Mourou, "Compression of amplified chirped optical pulses,” Opt. Commun. 56, 219-221 (1985).

2. A. Chong, L. Kuznetsova, and F. Wise, "Theoretical optimization of nonlinear chirped-pulse fiber amplifiers," J. Opt. Soc. Am. B 24, 1815-1823 (2007).

3. S. Kane, J. Squier, J. V. Rudd, and G. Mourou, "Hybrid grating-prism stretcher-compressor system with cubic phase and wavelength tenability and decreased alignment sensitivity," Opt. Lett. 19, 1876-1878 (1994).

4. D. C. O'Shea, "Group-velocity dispersion using commercial optical design programs," Appl. Opt. 45, 4740-4746 (2006).

5. U. Fuchs, U. D. Zeitner, and A. Tünnerman, "Ultrashort pulse propagation in complex optical systems," Opt. Express 13, 3852-3861 (2005).

6. A. G. Kostenbauder, "Ray-pulse matrices: a rational treatment for dispersive optical systems," IEEE J. Quantum Electron. 26, 1148-1157 (1990).

7. O. E. Martinez, "Matrix formalism for pulse compressors," IEEE J. Quantum Electron. 24, 2530-2536 (1988).

8. Y. Nabekawa and K. Midorikawa, "High-order pulse front tilt caused by high-order angular dispersion," Opt. Express 11, 3365-3376 (2003).

9. P. Gabolde, D. Lee, S. Akturk, and R. Trebino, "Describing first-order spatio-temporal distortions in ultrashort pulses using normalized parameters," Opt. Express 15, 242-251 (2007).

10. R. E. Sherriff, "Analytic expressions for group-delay 
dispersion and cubic dispersion in arbitrary prism sequences," J. Opt. Soc. Am. B 15, 1224-1230 (1997).

11. Y. Zaouter, D. N. Papadopoulos, M. Hanna, F. Druon, E. Cormier, and P. Georges, "Third-order spectral phase compensation in parabolic pulse compression," Opt. Express 15, 9372-9377 (2007).

12. S. Kane and J. Squier, "Grism-pair stretcher-compressor system for simultaneous second- and third-order dispersion compensation in chirped-pulse amplification," J. Opt. Soc. Am. B 14, 661-665 (1997).

13. E. A. Gibson, D. M. Gaudiosi, H. C. Kapteyn, R. Jimenez, S. Kane, R. Huff, C. Durfee, and J. Squier, "Efficient reflection grisms for pulse compression and dispersion compensation of femtosecond pulses," Opt. Lett. 31, 3363-3365 (2006).

14. J. R. Buckley, S. W. Clark, and F. W. Wise, "Generation of ten-cycle pulses from an ytterbium fiber laser with cubic phase compensation," Opt. Lett. 31, 1340-1342 (2006).

15. L. Kuznetsova, F. W. Wise, S. Kane, and J. Squier,
"Chirped-pulse amplification of femtosecond pulses in a Yb-doped fiber amplifier near the gain narrowing limit using a reflection grism compressor," presented at the Advanced Solid-State Photonics Topical Meeting and Tabletop Exhibit, Vancouver, Canada, January 28-31, 2007, paper TuB3.

16. G. Cheriaux, P. Rousseau, F. Salin, J. P. Chambaret, B. Walker, and L. F. Dimauro, "Aberration-free stretcher design for ultrashort-pulse amplification," Opt. Lett. 21, 414-416 (1996).

17. J. Jiang, Z. Zhang, and T. Hasama, "Evaluation of chirpedpulse-amplification systems with Offner triplet telescope stretchers," J. Opt. Soc. Am. B 19, 678-683 (2002).

18. F. Verluise, V. Laude, Z. Cheng, C. Spielmann, and P Tournois, "Amplitude and phase control of ultrashort pulses by use of an acousto-optic programmable dispersive filter: pulse compression and shaping," Opt. Lett. 25, $575-577$ (2000). 